\title{
Identification of two novel mammographic density loci at 6Q25.1
}

Judith S Brand ${ }^{1 *}$, Jingmei Li ${ }^{1}$, Keith Humphreys ${ }^{1}$, Robert Karlsson ${ }^{1}$, Mikael Eriksson ${ }^{1}$, Emma Ivansson ${ }^{1,2}$,

Per Hall ${ }^{1}$ and Kamila Czene ${ }^{1}$

\begin{abstract}
Introduction: Mammographic density (MD) is a strong heritable and intermediate phenotype for breast cancer, but much of its genetic variation remains unexplained. We performed a large-scale genetic association study including 8,419 women of European ancestry to identify MD loci.
\end{abstract}

Methods: Participants of three Swedish studies were genotyped on a custom Illumina iSelect genotyping array and percent and absolute mammographic density were ascertained using semiautomated and fully automated methods from film and digital mammograms. Linear regression analysis was used to test for SNP-MD associations, adjusting for age, body mass index, menopausal status and six principal components. Meta-analyses were performed by combining $P$ values taking sample size, study-specific inflation factor and direction of effect into account.

Results: Genome-wide significant associations were observed for two previously identified loci: ZNF365 (rs10995194, $P=2.3 \times 10^{-8}$ for percent MD and $P=8.7 \times 10^{-9}$ for absolute MD) and AREG (rs10034692, $P=6.7 \times 10^{-9}$ for absolute MD). In addition, we found evidence of association for two variants at $6 q 25.1$, both of which are known breast cancer susceptibility loci: rs9485370 in the TAB2 gene ( $P=4.8 \times 10^{-9}$ for percent MD and $P=2.5 \times 10^{-8}$ for absolute MD) and rs60705924 in the CCDC170/ESR1 region ( $P=2.2 \times 10^{-8}$ for absolute MD). Both regions have been implicated in estrogen receptor signaling with TAB2 being a potential regulator of tamoxifen response.

Conclusions: We identified two novel MD loci at 6q25.1. These findings underscore the importance of $6 q 25.1$ as a susceptibility region and provide more insight into the mechanisms through which MD influences breast cancer risk.

\section{Introduction}

Mammographic density (MD) reflects the amount of radiographically dense tissue on an X-ray of the breast (mammogram) and is an intermediate phenotype for breast cancer [1]. MD is highly heritable $\left(h^{2}=0.60-0.65\right)$ [2-5] and genetic loci associated with MD can provide insight into the biological mechanisms leading to breast cancer, which may serve as targets for treatment and preventive strategies [6]. Despite the high heritability, a large proportion of the genetic variation of MD remains unexplained [7-9]. The Marker of Density (MODE) consortium recently identified nine loci (AREG, ESR1, ZNF365, LSP1/TNNT3, IGF1, TMEM184B, SGSM3/MKL1, PRDM6, 8p11.23) associated with area-based MD $[7,8]$ as obtained with the semiautomated thresholding method

\footnotetext{
* Correspondence: Judith.brand@ki.se

'Department of Medical Epidemiology and Biostatistics, Karolinska Institutet, Nobels Väg 12A, 17177 Stockholm, Sweden

Full list of author information is available at the end of the article
}

Cumulus [10]. Although Cumulus is still regarded as the 'gold standard' for screen-film mammography, fully automated methods may help in the identification of additional variants as these methods are less prone to random measurement error. We performed a large-scale genetic association study combining semiautomated and fully automated density measures to identify novel MD loci.

\section{Methods}

\section{Study participants}

For the present study, we included participants of European ancestry from three Swedish studies: KARolinska MAmmography project for risk prediction of breast cancer (KARMA), Linné-bröst 1 (LIBRO-1) and the Singapore and Sweden Breast Cancer Study (SASBAC). KARMA is a prospective screening-based study initiated in January 2011 and includes 70,877 women who attended mammography screening or clinical mammography at four hospitals in Sweden. In 2010, a random sample of 5,531 cancer-free 
women was genotyped of whom 4,025 had raw digital mammograms stored. LIBRO-1 is a breast cancer cohort including 5,715 malignant cases diagnosed between 2001 and 2008 in the Stockholm/Gotland area. The majority of the cohort $(\mathrm{N}=5,125)$ was genotyped and prediagnostic film mammograms were successfully retrieved for 2,805 women. A further 1,589 women were drawn from the SASBAC study, which is a population-based case-control study including postmenopausal breast cancer cases in Sweden aged 50 to 74 years at time of enrollment (1 October 1993 to 31 March 1995) and age-matched controls. Ethical approval of KARMA, LIBRO-1 and SASBAC was given by the ethical review board at Karolinska Institutet (Stockholm, Sweden) and written informed consent was obtained from all participants.

\section{Assessment of mammographic density}

Mammographic density was obtained from the mediolateral oblique (MLO) view in all three studies using different measurement tools. In KARMA, MD was estimated from raw digital mammograms using a volumetric method (Volpara) [11]. Volpara shows good agreement with breast magnetic resonance imaging (MRI) data [11] and its measures (percent and absolute dense volume) have been validated as being predictive of breast cancer risk $[12,13]$. In SASBAC and LIBRO-1, MD was estimated using an area-based method from film mammograms with respectively Cumulus [10] and an automated algorithm based on the image processing software ImageJ that mimics Cumulus [14, 15]. ImageJ shows good agreement with Cumulus (the 'gold standard' for film mammography) with high levels of correlation for both percent and absolute dense area [13, 16, 17].

Since volumetric measures incorporate information on breast thickness, the underlying distribution of areabased and volumetric measures are slightly different, with the latter being more right-skewed with a smaller range of possible values (Figure S1 in Additional file 1).

\section{Genotyping and imputation}

All women were genotyped using the custom Ilumina iSelect genotyping array of the Collaborative Oncological Gene-environment Study (iCOGS) which comprises 211,155 single nucleotide polymorphisms (SNPs) primarily selected for replication of loci putatively associated with breast cancer and other cancers [18]. Details of the iCOGS array design, sample handling and postgenotyping quality control (QC) processes are described in depth elsewhere [18]. In brief, samples were excluded from analysis for any of the following reasons: low or high heterozygosity, individuals not concordant with previous genotyping, discordant duplicate pairs and first-degree relatives. Standard SNP QC was performed in Plink (version 1.07) [19] and SNPs with minor allele frequency (MAF) $<0.01$ or deviation from Hardy-Weinberg equilibrium (HWE) at $P<1 \times 10^{-6}$ in controls or $P$ $<1 \times 10^{-12}$ in cases were excluded, leaving 170,798 SNPs for the combined analyses. To increase resolution and coverage for regional association testing, nongenotyped SNPs were imputed using the 1000 Genomes Project March 12 release as a reference [20]. Data were imputed in a two-stage procedure, using SHAPEIT to derive phased genotypes and IMPUTE version 2 (IMPUTEv2) to perform the imputation on the phased data [21]. The imputation was performed using $5 \mathrm{Mb}$ nonoverlapping windows across the whole genome. Postimputation quality control was based on the IMPUTE info score and SNPs with a score $\leq 0.80$ or MAF $<0.01$ were excluded.

\section{Statistical analyses}

SNP association analysis was performed separately within each study. Genotyped SNPs were analysed in Plink (version 1.07) [19] using linear regression and assuming an additive genetic model. We analyzed three MD phenotypes: percent density (percent MD), absolute dense tissue (absolute MD) and the absolute nondense tissue. Since volumetric mammographic measures follow a different distribution than area-based measures, different types of transformation were used to approximate the normal distribution (log-transformation for volumetric measures and square-root transformation for area-based measures) (Figure S1 in Additional file 1).

Differences in study design and measurement technique did not allow us to perform meta-analyses based on study-specific effect estimates (beta coefficients). Instead, we performed meta-analyses combining studyspecific $P$ values in METAL (25 March 2011 release) [22] taking sample size, study-specific inflation factor and direction of effect into account.

Regional association plots were generated using LocusZoom with the $400 \mathrm{~kb}$ region centered on the index SNP [23]. Imputed SNPs within the region were analyzed with SNPTEST (version 2.5.2) [24, 25] based on the score test, which uses allele dosages instead of genotype calls.

Population stratification was assessed using principal component (PC) analysis in EIGENSTRAT (version 3.0) [26, 27]. All analyses were adjusted for age (years), body mass index (BMI) $\left(\mathrm{kg} / \mathrm{m}^{2}\right)$, menopausal status (postmenopausal vs premenopausal) and six study-specific PC scores to account for population substructure.

\section{Functional annotation and breast cancer association analysis of identified variants}

Functional annotation of associated variants and their proxies ( $\mathrm{r}^{2} \geq 0.8$ in 1000 Genomes CEU population) was performed using the HaploReg v2 software [28]. We 
studied putative functional variants using data from the Encyclopedia of DNA Elements (ENCODE) project [29], in particular the chromatin state segmentation [30] for the human mammary epithelial cells (HMEC) cell line.

We checked for associations between associated variants and breast cancer risk by doing a lookup in the Breast Cancer Association Consortium (BCAC) including a total of 55,540 breast cancer cases and 51,168 controls with iCOGS genotyping data. We also verified associations with MD loci that were previously identified by the genome-wide association study (GWAS) coordinated by MODE [8]. These analyses were performed in KARMA and LIBRO-1 participants only, as SASBAC is part of the MODE consortium.

\section{Results}

Table 1 summarizes the characteristics of the study participants in each individual study. Most participants were postmenopausal: $76.1 \%$ in LIBRO-1 and $100 \%$ in SASBAC, with a mean age of 58.4 years and 62.4 years respectively. Participants of KARMA were slightly younger (mean age $=53.6$ years), with a larger contribution of premenopausal women (51.0\%). No substantial difference in BMI was observed across the individual studies.

Quantile-quantile (QQ) plots for each MD phenotype are shown in Figure S2 in Additional file 2.

All plots displayed no global departure from the expected null distribution of $P$ values and the genetic inflation factor $(\lambda)$ was $1.005,1.015$ and 1.024 for KARMA, LIBRO-1 and SASBAC respectively, indicating that residual confounding by population stratification is negligible.

Figure S3 (Additional file 3) shows the Manhattan plots displaying the $\log 10$-transformed $P$ values for each genotyped SNP per MD phenotype. In total, we identified two loci for percent MD (TAB2, ZNF365) and four loci for absolute MD (AREG, TAB2, CCDC170/ESR1, ZNF365) (Table 2; Fig. 1). Two of the loci (ZNF565, AREG) were recently identified by MODE $[7,8]$, but the loci mapping to 6q25.1 (TAB2 and CCDC170/ESR1) have not been reported previously as being associated with $\mathrm{MD}$ at a genome-wide significance level. No significant associations were observed for the absolute nondense tissue (Figure S3 in Additional file 3).

The strongest association at $6 \mathrm{q} 25.1$ was found for rs9485370 in the TGF-beta-activated kinase 1/MAP3K7binding (TAB2) gene, where each additional copy of the minor allele was associated with a decrease in percent MD $\left(P=4.8 \times 10^{-9}\right)$ and absolute MD $\left(P=6.7 \times 10^{-9}\right)$ (Table 2, Fig. 1). Rs9485370 is located in a putative enhancer element in HMEC cell lines and is linked to a number of SNPs which have been predicted to influence transcription factor binding (Figure S4 in Additional file 4).

The minor allele frequency (MAF) of rs9485370 varies widely across populations with the effect allele $(\mathrm{T})$ being more common in Asians than Europeans. Rs9485370 is not an established breast cancer SNP in women of European ancestry, but an SNP in complete linkage disequilibrium (LD) (rs9485372; $\mathrm{r}^{2}=1, \mathrm{D}^{\prime}=1$ ) has previously been associated with breast cancer risk in East Asian women [31, 32]. Rs9485370 did not reach genome-wide significance in $\mathrm{BCAC}$, but there was evidence of a stronger association in Asian (odds ratio $(\mathrm{OR})=0.89, P=7.4 \times 10^{-6}$ ) than in European women $\left(\mathrm{OR}=0.96, P=1.4 \times 10^{-3}\right)$ and the direction of association was consistent with the effect of MD on breast cancer risk (Table S1 in Additional file 5). All associated SNPs in the $400 \mathrm{~kb}$ window were in LD with s9485370 and there was no evidence of additional independent signals in this locus (Fig. 1).

The second hit at 6q25.1 was rs60705924, located $14 \mathrm{~kb}$ downstream of $C C D C 170$ and $22 \mathrm{~kb}$ upstream of ESR1. Each minor allele at rs60705924 was associated with an increase in absolute $\mathrm{MD}\left(P=2.2 \times 10^{-8}\right)$ (Table 2, Fig. 1) but the association was weaker and nonsignificant for percent MD $\left(P=1.2 \times 10^{-4}\right)$. Rs60705924 is strongly correlated with breast cancer SNP rs2046210 $\left(\mathrm{r}^{2}=0.89, \mathrm{D}^{\prime}=1.00\right)$ [18] and its association with breast cancer $(\mathrm{OR}$ in $\mathrm{BCAC}$ European sample $=1.08$, $P=1.9 \times 10^{-13}$ ) follows the same direction as its association with absolute MD (Table S1 in Additional file 5). Three SNPs in strong LD with rs60705924 (rs7763637, rs6557160, rs6913578) map to promoter/enhancer histone marks in HMEC cell lines (Table S4 in Additional file 4). Rs60705924 is also in proximity to rs12665607, a SNP that

Table 1 Descriptive characteristics of the studies included

\begin{tabular}{|c|c|c|c|c|c|c|c|c|c|}
\hline \multirow[t]{2}{*}{ Study } & \multirow[t]{2}{*}{ Number } & \multirow[t]{2}{*}{ Mammogram } & \multirow[t]{2}{*}{ Measure } & \multirow{2}{*}{$\begin{array}{l}\begin{array}{l}\text { Age } \\
\text { (years) } \\
\text { Mean (SD) }\end{array}\end{array}$} & \multirow{2}{*}{$\begin{array}{l}\mathrm{BMl} \\
\left(\mathrm{kg} / \mathrm{m}^{2}\right) \\
\text { Mean (SD) }\end{array}$} & \multirow{2}{*}{$\begin{array}{l}\text { Post- } \\
\text { menopause } \\
\text { Percent (N) }\end{array}$} & \multirow{2}{*}{$\begin{array}{l}\text { Percent } \\
\text { density }(\%)^{\mathrm{a}} \\
\text { Median (IQR) }\end{array}$} & \multirow{2}{*}{$\begin{array}{l}\text { Absolute dense }^{a} \\
\text { Median (IQR) }\end{array}$} & \multirow{2}{*}{$\begin{array}{l}\text { Absolute nondense } \\
\text { Median (IQR) }\end{array}$} \\
\hline & & & & & & & & & \\
\hline KARMA & 4025 & Raw digital & Volpara & $53.6(9.4)$ & $25.3(4.2)$ & $51.0(2,054)$ & $8.4(6.5)$ & $60.4(36.8)$ & $677(581)$ \\
\hline SASBAC & 1589 & Digitized screen-film & Cumulus & $62.4(6.4)$ & $25.6(3.8)$ & $100(1,589)$ & $11.8(17.7)$ & $18.1(26.2)$ & $140(76)$ \\
\hline LIBRO-1 & 2805 & Digitized screen-film & ImageJ & $58.4(8.8)$ & $25.3(4.0)$ & $76.1(2,134)$ & $30.0(21.6)$ & $30.6(21.5)$ & $71(30)$ \\
\hline
\end{tabular}

All mammograms were from the mediolateral oblique (MLO) view

$B M I$ body mass index, $S D$ standard deviation, $I Q R$ interquartile range

apercent density (percent MD) in \%; absolute dense tissue (absolute MD) in $\mathrm{cm}^{3}$ (KARMA) and $\mathrm{cm}^{2}$ (SASBAC and LIBRO-1); absolute nondense tissue in $\mathrm{cm}^{3}$ (KARMA) and $\mathrm{cm}^{2}$ (SASBAC and LIBRO-1) 
Table 2 Single nucleotide polymorphisms associated with percent and absolute mammographic density

\begin{tabular}{|c|c|c|c|c|c|c|c|c|c|c|c|c|c|c|c|}
\hline \multirow[b]{2}{*}{$\mathrm{CHR}$} & \multirow[b]{2}{*}{ SNP } & \multirow[b]{2}{*}{$\mathrm{BP}$} & \multirow[b]{2}{*}{ Genes } & \multirow[b]{2}{*}{ Alleles $^{a}$} & \multicolumn{3}{|c|}{ KARMA } & \multicolumn{3}{|c|}{ SASBAC } & \multicolumn{5}{|c|}{ LIBRO-1 } \\
\hline & & & & & MAF & beta (se) & $P$ & MAF & beta (se) & $P$ & MAF & beta (se) & $P$ & $\begin{array}{l}P \\
\text { overall }\end{array}$ & $\begin{array}{l}P \\
\text { het }\end{array}$ \\
\hline \multicolumn{16}{|c|}{ Percent density } \\
\hline 6 & rs9485370 & 149606801 & $T A B 2$ & $\mathrm{G} / \mathrm{T}$ & 0.18 & $-0.05(0.01)$ & $2.3 \times 10^{-5}$ & 0.17 & $-0.16(0.08)$ & 0.04 & 0.17 & $-0.16(0.05)$ & $3,9 \times 10^{-4}$ & $4.8 \times 10^{-9}$ & 0.88 \\
\hline $10^{\mathrm{b}}$ & rs10995194 & 64288130 & ZNF365 & $\mathrm{G} / \mathrm{C}$ & 0.16 & $-0.05(0.01)$ & $7.1 \times 10^{-6}$ & 0.15 & $-0.13(0.08)$ & 0.10 & 0.15 & $-0.15(0.05)$ & $1.9 \times 10^{-3}$ & $2.3 \times 10^{-8}$ & 0.62 \\
\hline \multicolumn{16}{|c|}{ Absolute dense } \\
\hline $4^{b}$ & rs10034692 & 75419787 & AREG & $\mathrm{A} / \mathrm{G}$ & 0.32 & $-0.04(0.01)$ & $9.6 \times 10^{-5}$ & 0.31 & $-0.23(0.08)$ & $6.1 \times 10^{-3}$ & 0.31 & $-0.14(0.04)$ & $5.5 \times 10^{-4}$ & $6.7 \times 10^{-9}$ & 0.96 \\
\hline 6 & rs9485370 & 149606801 & TAB2 & $\mathrm{G} / \mathrm{T}$ & 0.18 & $-0.04(0.01)$ & $2.0 \times 10^{-3}$ & 0.17 & $-0.22(0.10)$ & 0.03 & 0.17 & $-0.22(0.05)$ & $1.0 \times 10^{-5}$ & $2.5 \times 10^{-8}$ & 0.37 \\
\hline 6 & rs60705924 & 151955985 & CCDC170-ESR1 & $A / G$ & 0.31 & $0.04(0.01)$ & $1.9 \times 10^{-4}$ & 0.31 & $0.33(0.08)$ & $3.0 \times 10^{-5}$ & 0.31 & $0.09(0.04)$ & 0.03 & $2.2 \times 10^{-8}$ & 0.13 \\
\hline $10^{\mathrm{b}}$ & rs10995194 & 664288130 & ZNF365 & $\mathrm{G} / \mathrm{C}$ & 0.16 & $-0.07(0.01)$ & $1.1 \times 10^{-6}$ & 0.15 & $-0.21(0.10)$ & 0.05 & 0.15 & $-0.15(0.05)$ & $5.1 \times 10^{-3}$ & $8.7 \times 10^{-9}$ & 0.55 \\
\hline
\end{tabular}

\section{Genes refer to genes and nearby genes}

MD mammographic density, CHR chromosome, SNP single nucleotide polymorphism, BP base pair position (NCBI Build 37), MAF minor allele frequencies

$P$ overall $=P$ value meta-analysis; $P$ het $=P$ value chi-square test for heterogeneity

${ }^{\mathrm{a}}$ Major allele (reference allele)/minor allele (effect allele)

b Previously identified in the Marker of Density (MODE) consortium: $R^{2}$ rs10995194 and MODE SNP rs10995190 $=1$ (1000 Genomes Project, Pilot 1 (CEU)) 
A

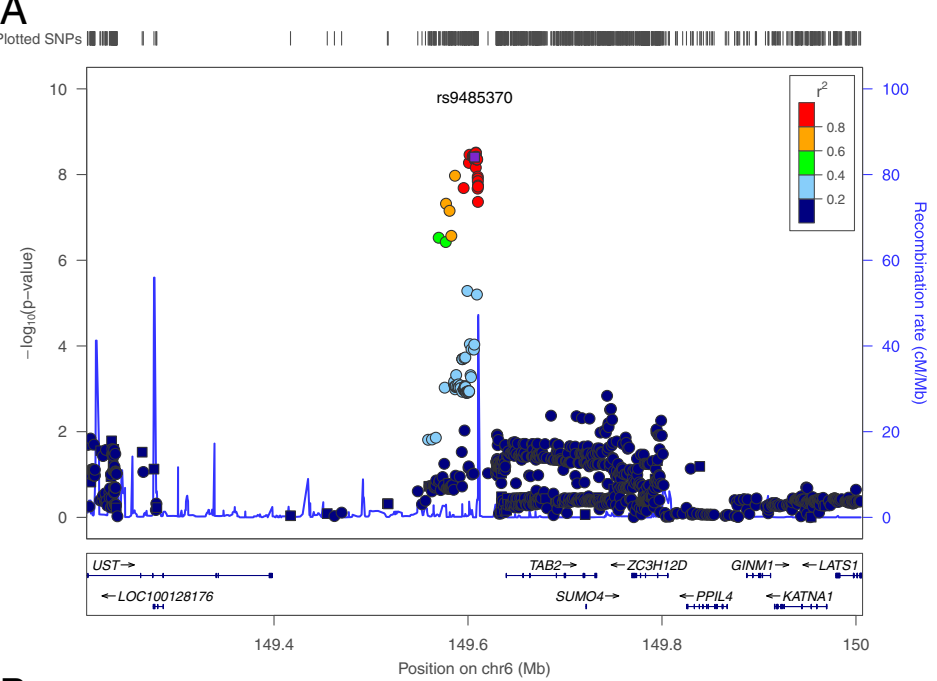

B

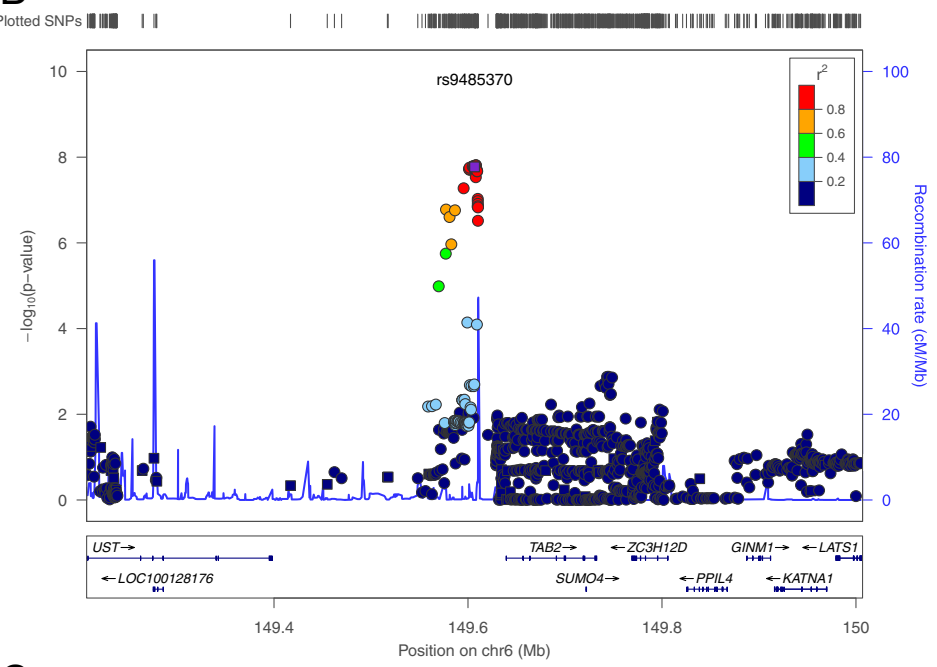

C

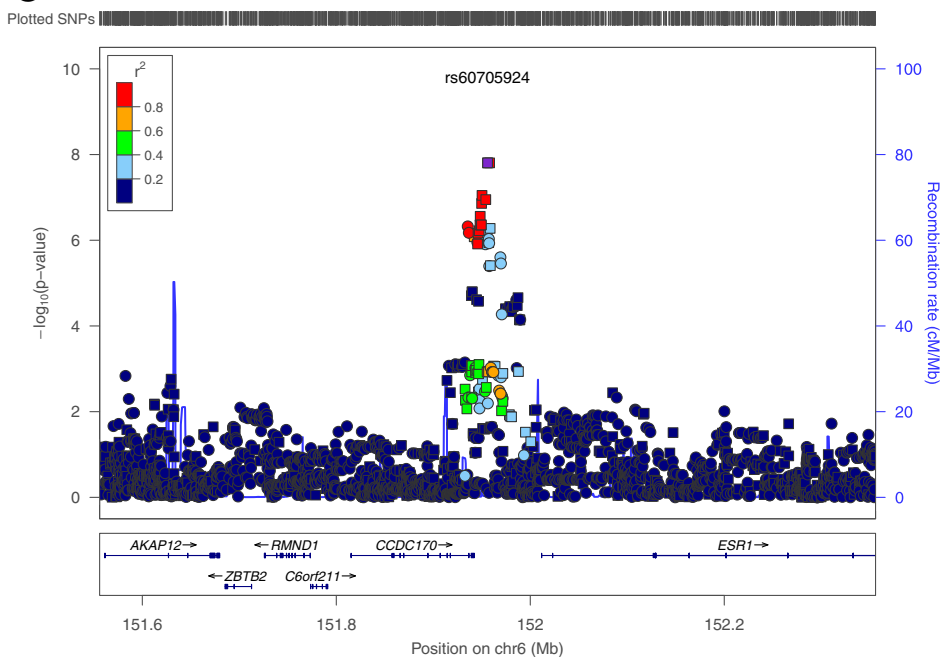

Fig. 1 (See legend on next page.) 
(See figure on previous page.)

Fig. 1 Regional plots of SNPs associated with percent and absolute mammographic density. $\mathbf{a}=$ regional association plot rs 9485370 for percent density; (b) = regional association plot rs9485370 for absolute dense tissue; (c) = regional association plot rs60705924 for absolute dense tissue. Plot shows - $\log 10 P$ values (y-axis) by chromosomal position (x-axis). Top genotyped SNPs (rs9485370 and rs60705924) are shown in purple. Squares denote genotyped SNPs; circles denote imputed SNPs. Colors indicate the extent of linkage disequilibrium with rs9485370 and rs60705924. Genetic recombination rates are estimated using 1000 Genomes EUR sample and are shown with the light blue line. Physical positions are based on NCBI build37 of the human genome. Note: rs9485370 falls within transcript ENST00000536230 of TAB2, which spans chr6:149539777-149731075. The plot was generated using LocusZoom software. SNP single nucleotide polymorphism

was recently found to be associated with absolute dense area in MODE [8] and highly correlated with breast cancer SNP rs12662670 $\left(\mathrm{r}^{2}=0.89 ; \mathrm{D}^{\prime}=1.00\right)$. However, rs60705924 and rs12665607 are only weakly correlated $\left(\mathrm{r}^{2}=0.19 ; \mathrm{D}^{\prime}=1.00\right)$ and the association with rs60705924 was only slightly attenuated in conditional analysis $\left(P=4.8 \times 10^{-6}\right)$. Regional association analysis revealed no additional independent SNPs in the $400 \mathrm{~kb}$ window of rs60705924 (Fig. 1).

We also tested for associations with MD loci that were previously identified in GWAS coordinated by MODE. We could confirm associations with the majority of loci found by MODE (Table S2 in Additional file 6), except for LSP1 $(P=0.25$ for percent MD), TMEM184B $(P=0.14$ for percent $\mathrm{MD}$ and $P=0.30$ for absolute dense tissue) and rs7816345 at chromosome $8(P=0.31$ for percent $\mathrm{MD})$, although there was some evidence of an association between TMEM184B and volumetric MD in KARMA. Although no SNPs reached genome-wide significance in our meta-analysis of absolute nondense tissue, we could replicate the nondense locus (rs7816345) that was recently identified by $\operatorname{MODE}\left(P=2.4 \times 10^{-4}\right)$ (Table S2 in Additional file 6).

\section{Discussion}

We performed a meta-analysis of three large-scale genetic association studies to identify novel MD loci. Using semiautomated and fully automated measures, we were able to identify two additional variants at $6 \mathrm{q} 25.1$ (TAB2 and CCDC170/ESR1) that were associated with both volumetric and area-based MD. We also confirmed associations with several loci (ZNF365 and AREG) that were previously identified by MODE [8].

Like MODE, we identified more genetic loci for absolute than for percent MD. Our most significant hit was rs9485370 mapping to the TAB2 gene. This SNP has previously been associated with breast cancer risk in East Asian women [31, 32], but not with mammographic density at a genome-wide significance level. The protein encoded by the TAB2 gene is an important mediator of interleukin-1 (IL-1)-induced activation of the NFkB and MAPK8/JNK pathway [33] which has been associated with early tumorigenesis and metastasis [34, 35] as well as mammary development [36]. The TAB2 protein also interacts directly with the N-terminal domain of the estrogen receptor alpha (ESR1) and has been implicated in proinflammatory induced reactivation of repressed estrogen receptor (ER) signaling pathways [37, 38]. Because of its role in ER signaling, TAB2 is seen as a potential target for reversing tamoxifen resistance in breast cancer cells [38].

The second variant at 6q25.1 (rs60705924) is located in CCDC170/ESR1 region, a well-established breast cancer locus, but its putative functions are not well defined. Previous GWAS and candidate approaches have identified multiple genetic variants at CCDC170/ESR1 to be associated with breast cancer as well as mammographic density [39]. A breast cancer SNP in strong LD with rs60705924 and rs2046210 has previously been identified in candidate approaches of area-based and volumetric MD $[5,7]$, but not at genome-wide significance level. SNP rs2046210 is more strongly associated with ER-positive than -negative tumors $[40,41]$ and our data suggest that at least part of the association with breast cancer is mediated through mammographic density. Recent data further indicate that recurrent rearrangements between the ESR1 and $C C D C 170$ gene are linked to more aggressive and endocrine-resistant cancers [42]. Fine-mapping studies of 6q25.1 are needed to provide more insight into the independent and causal variants in this specific region.

To our knowledge, this is one of the largest studies analyzing genetic determinants of fully and semiautomated MD measures. All mammograms in the study were obtained from the MLO view and all participants were genotyped on the same genotyping platform, reducing the likelihood of measurement errors due to between-view and interassay differences. However, our findings need to be interpreted in light of the different MD methods used. First of all, we combined screen-film and digital mammograms in our meta-analysis. Previous studies have shown that MD measurements from digital mammograms tend to be lower than from film mammograms [43]. Furthermore, different measurement tools were used in each individual study. Both area-based and volumetric methods aim to quantify the amount of fibroglandular tissue in the breast from two-dimensional mammograms, but the measurement techniques used are slightly different. Area-based methods use an intrinsic threshold technique [either semiautomated (Cumulus) or fully automated (ImageJ)] to categorize pixels as dense or nondense, whereas Volpara is 
specifically designed to quantify the density in each individual pixel on a continuous scale while accounting for interindividual differences in breast thickness. Several studies have evaluated the agreement between Cumulus and ImageJ, all showing high correlation coefficients for percent dense area ( $r$ ranging from 0.88 to 0.92$)[13,16,17]$ and absolute dense area ( $r$ ranging from 0.89 to 0.90$)$ [13, 17]. High levels of agreement have also been reported for percent dense area and volume ( $r$ ranging from 0.86 to 0.93 ) $[12,13]$, and recent data confirm the good overall correlation between all three measures with similar breast cancer risk estimates for Cumulus, ImageJ and Volpara percent MD [13, 44]. The correlation between absolute dense area and volume is somewhat weaker ( $r$ ranging from 0.41 to $0.55)[12,13]$. Heritability estimates also tend to be lower for the absolute dense volume than for the absolute dense area [5], indicating that these absolute measures represent different aspects of MD. Since we cannot rule out the presence of area- and volumetric-specific MD loci, we could have missed SNPs that are associated with the absolute dense volume, but not with its area-based counterpart. This might also explain the lack of replication for some of the SNPs previously identified by MODE for area-based MD [8]. Future GWAS aimed at identifying genetic variants of both area-based and volumetric MD will provide more insight into this matter. Although our study might be limited in terms of power due to the combination of different $\mathrm{MD}$ methods (e.g., mammogram type and measurement technique), this could not have affected the validity of our findings. Of note, all SNP-MD associations were in the same direction with no evidence of between-study heterogeneity. As such, our study has identified loci that are associated with MD, regardless of measurement technique and mammogram type used.

Studies identifying MD loci are important to increase our understanding of the biological mechanisms leading to breast cancer in women with high mammographic density. Such insights come primarily from SNPs that are associated with both mammographic density and breast cancer risk $[12,45]$, including the variants identified in the present study. This information might also be relevant for identifying new targets for treatment and preventive strategies. Our results, for instance, highlight the importance of the 6q25.1 region in the etiology of breast cancer among women with dense breasts. From a clinical perspective, SNPs and downstream pathways that are associated with mammographic density, but not with breast cancer risk, are of limited value, as these SNP-MD associations are not likely to influence breast cancer as a disease endpoint.

\section{Conclusions}

In conclusion, we identified two novel MD loci at 6q25.1 in a large-scale genotyping effort of semiautomated and fully automated MD measures, which have previously been associated with breast cancer risk. These findings underscore the importance of $6 \mathrm{q} 25.1$ as a susceptibility region and provide more insight into the mechanisms through which MD influences breast cancer risk. Future large-scale genetic association studies of area-based and volumetric MD are needed to increase our understanding of the genetic basis of mammographic density and its link with breast cancer.

\section{Additional files}

Additional file 1: Figure S1. Distributions of mammographic density phenotypes, stratified by study. Distributions of mammographic measures before (A) and after transformation (B). Percent density in \%; absolute dense tissue in $\mathrm{cm}^{3}$ (KARMA) and $\mathrm{cm}^{2}$ (SASBAC and LIBRO-1); absolute nondense tissue in $\mathrm{cm}^{3}$ (KARMA) and $\mathrm{cm}^{2}$ (SASBAC and LIBRO-1). Volumetric mammographic measures were log-transformed (KARMA) and area-based mammographic measures were square-root-transformed (SASBAC and LIBRO-1) prior to analyses.

Additional file 2: Figure S2. Quantile-quantile (QQ) plots, per mammographic density phenotype. $\mathrm{A}=$ percent density; $\mathrm{B}=$ absolute dense tissue; $C=$ absolute nondense tissue. The observed $P$ values based the on meta-analysis of KARMA, SASBAC and LIBRO-1 are plotted against the expected distribution of $P$ values under the null distribution.

Additional file 3: Figure S3. Manhattan plots of the combined association results, per mammographic density phenotype. $\mathrm{A}=$ percent density; $\mathrm{B}=$ absolute dense tissue; $\mathrm{C}=$ absolute nondense tissue. The $-\log _{10}$ $(P)$ values are plotted against chromosomal base-pair position. Genome-widesignificant hits $\left(P<5 \times 10^{-8}\right)$ are indicated in red.

Additional file 4: Figure S4. Annotation of rs9485370 and rs60705924 by their effect on regulatory motifs according to the HaploREG database. $A=r s 9485370 ; B=r s 60705924$

Additional file 5: Table S1. Associations between genome-widesignificant SNPs and breast cancer risk in the Breast Cancer Association Consortium (BCAC).

Additional file 6: Table S2. Replication analysis of SNPs identified by MODE, per mammographic density phenotype.

\section{Abbreviations}

AREG: amphiregulin; BCAC: Breast Cancer Association Consortium; BMI: body mass index; CCDC170: coiled-coil domain containing 170;

ENCODE: Encyclopedia of DNA Elements; ER: estrogen receptor; ESR1: estrogen receptor 1; GWAS: genome-wide association study; HMEC: human mammary epithelial cells; HWE: Hardy-Weinberg equilibrium; iCOGS: Ilumina iSelect genotyping array of the Collaborative Oncological Gene-environment Study; IGF1: insulin-like growth factor 1; IL-1: interleukin-1; IMPUTEV2: IMPUTE version 2; KARMA: KARolinska MAmmography project for risk prediction of breast cancer; LD: linkage disequilibrium; LIBRO-1: Linné-bröst 1; LSP1: Iymphocyte- specific protein 1; MAF: minor allele frequency; MAPK8/JNK: mitogen-activated protein kinase 8/C-Jun N-terminal kinase; MD: mammographic density; MKL1: MKL/myocardin-like protein 1; MLO: mediolateral oblique; MODE: the Marker of Density consortium; NFkB: nuclear factor kappa B; OR: odds ratio; PRDM6: PR domain containing 6; QC: quality control; QQ: quantile-quantile; SASBAC: the Singapore and Sweden Breast Cancer study; SGSM3: small G protein signaling modulator 3; SNP: single nucleotide polymorphism; TAB2: TGF-beta-activated kinase 1/MAP3K7-binding protein 2; TMEM184B: transmembrane protein 184b; TNNT3: troponin T type 3; ZNF365: zinc finger protein 365.

\section{Competing interests}

The study sponsors had no role in the design of the study, the collection, analysis or interpretation of the data, the writing of the manuscript or the decision to submit the manuscript for publication. All authors declare that they have no conflict of interest. 


\section{Authors' contributions}

$J S B, J L, K H, P H$ and $K C$ conceived and designed the study. ME contributed to the acquisition of the data. JSB performed the statistical analyses with assistance from $J \mathrm{~L}, \mathrm{KH}, \mathrm{RK}$ and El. All authors contributed to the interpretation of the data. JSB drafted the manuscript in close collaboration with all authors. All authors critically revised and edited the manuscript and approved the final version to be submitted for publication. All authors are accountable for the accuracy and integrity of the work.

\section{Acknowledgements}

The authors thank BCAC for sharing breast cancer association results for selected SNPS and the investigators of the iCOGS project. The iCOGS project would not have been possible without the contributions of the following: Paul Pharoah, Kyriaki Michailidou, Manjeet K. Bolla, Qin Wang (BCAC), Andrew Berchuck (OCAC), Rosalind A. Eeles, Ali Amin Al Olama, Zsofia Kote-Jarai, Sara Benlloch (PRACTICAL), Georgia Chenevix-Trench, Antonis Antoniou, Lesley McGuffog and Ken Offit (CIMBA), Joe Dennis, Alison M. Dunning, Andrew Lee, and Ed Dicks, Craig Luccarini and the staff of the Centre for Genetic Epidemiology Laboratory, Javier Benitez, Anna Gonzalez-Neira and the staff of the CNIO genotyping unit, Jacques Simard and Daniel C. Tessier, Francois Bacot, Daniel Vincent, Sylvie LaBoissière and Frederic Robidoux and the staff of the McGill University and Génome Québec Innovation Centre, Stig E. Bojesen, Sune F. Nielsen, Borge G. Nordestgaard, and the staff of the Copenhagen DNA laboratory, and Julie M. Cunningham, Sharon A. Windebank, Christopher A. Hilker, Jeffrey Meyer and the staff of Mayo Clinic Genotyping Core Facility. Funding for the iCOGS infrastructure came from: the European Community's Seventh Framework Programme under grant agreement no 223175 (HEALTH-F2-2009-223175) (COGS), Cancer Research UK (C1287/A10118, C1287/A 10710, C12292/A11174, C1281/A12014, C5047/A8384, C5047/A15007, C5047/A10692), the National Institutes of Health (CA128978) and Post-Cancer GWAS initiative (1U19 CA148537, 1 U19 CA148065 and 1 U19 CA148112 - the GAME-ON initiative), the Department of Defence (W81XWH-10-1-0341), the Canadian Institutes of Health Research (CIHR) for the CIHR Team in Familial Risks of Breast Cancer, Komen Foundation for the Cure, the Breast Cancer Research Foundation, and the Ovarian Cancer Research Fund. We also thank Dr. Ralph Highnam for providing technical support and assistance on the Volpara software for volumetric mammographic density and all participants who took part in the individual studies and all staff who have enabled this work to be carried out.

\section{Funding}

This work was financed by the Swedish Research Council (grant no: 20142271), Swedish Cancer Society (grant no: CAN 2013/469) and Cancer Society in Stockholm (grant no: 141092). The KARMA study is supported by the Märit and Hans Rausing Initiative Against Breast Cancer and the Cancer and Risk Prediction Center (CRisP), a Linneus Centre (grant no: 70867902) financed by the Swedish Research Council. Jingmei Li is a UNESCO-L'OREAL International Fellow. K. Humphreys is supported by the Swedish Research Council (grant no: 521-2011-3205). The breast cancer meta-analysis is supported by the GAME-ON DRIVE (grant no: CA148065) and BCAC initiatives.

\section{Author details}

${ }^{1}$ Department of Medical Epidemiology and Biostatistics, Karolinska Institutet, Nobels Väg 12A, 17177 Stockholm, Sweden. Swedish eScience Research Centre (SeRC), Karolinska Institutet, Nobels Väg 12A, 17177 Stockholm, Sweden.

Received: 23 January 2015 Accepted: 19 May 2015 Published online: 03 June 2015

\section{References}

1. Boyd NF, Guo H, Martin LJ, Sun L, Stone J, Fishell E, et al. Mammographic density and the risk and detection of breast cancer. N Engl J Med. 2007;356:227-36.

2. Boyd NF, Dite GS, Stone J, Gunasekara A, English DR, McCredie MR, et al. Heritability of mammographic density, a risk factor for breast cancer. N Engl J Med. 2002:347:886-94.

3. Stone J, Dite GS, Gunasekara A, English DR, McCredie MR, Giles GG, et al. The heritability of mammographically dense and nondense breast tissue. Cancer Epidemiol Biomarkers Prev. 2006;15:612-7.
4. Varghese JS, Thompson DJ, Michailidou K, Lindstrom S, Turnbull C, Brown J, et al. Mammographic breast density and breast cancer: evidence of a shared genetic basis. Cancer Res. 2012;72:1478-84.

5. Brand JS, Humphreys K, Thompson DJ, Li J, Eriksson M, Hall P, et al. Volumetric mammographic density: heritability and association with breast cancer susceptibility Loci. J Natl Cancer Inst. 2014;106. doi:10.1093/jnci/dju334. Print 2014 Dec

6. Boyd NF, Rommens JM, Vogt K, Lee V, Hopper JL, Yaffe MJ, et al. Mammographic breast density as an intermediate phenotype for breast cancer. Lancet Oncol. 2005;6:798-808.

7. Lindstrom S, Vachon CM, Li J, Varghese J, Thompson D, Warren R, et al. Common variants in ZNF365 are associated with both mammographic density and breast cancer risk. Nat Genet. 2011;43:185-7.

8. Lindstrom S, Thompson DJ, Paterson AD, Li J, Gierach GL, Scott C, et al. Genome-wide association study identifies multiple loci associated with both mammographic density and breast cancer risk. Nat Commun. 2014;5:5303.

9. Fernandez-Navarro P, Gonzalez-Neira A, Pita G, Diaz-Uriarte R, Tais Moreno L, Ederra $\mathrm{M}$, et al. Genome-wide association study identifies a novel putative mammographic density locus at 1q12-q21. Int J Cancer. 2015;136:2427-36.

10. Byng JW, Boyd NF, Fishell E, Jong RA, Yaffe MJ. The quantitative analysis of mammographic densities. Phys Med Biol. 1994;39:1629-38.

11. Highnam R, Brady M, Yaffe M, Karssemeijer N, Harvey J. Robust breast composition measurement - Volpara ${ }^{\text {TM }}$. Lect Notes Comput Sci. 2010;6136:342-9.

12. Brand JS, Czene K, Shepherd JA, Leifland K, Heddson B, Sundbom A, et al. Automated measurement of volumetric mammographic density: a tool for widespread breast cancer risk assessment. Cancer Epidemiol Biomarkers Prev. 2014;23:1764-72.

13. Eng A, Gallant Z, Shepherd J, McCormack V, Li J, Dowsett M, et al. Digital mammographic density and breast cancer risk: a case-control study of six alternative density assessment methods. Breast Cancer Res. 2014;16:439. doi:10.1186/s13058-014-0439-1.

14. ImageJ: U.S. National Institutes of Health. Bethesda, MD, USA. http://imagej.nih.gov/ij/

15. Sovio U, Li J, Aitken Z, Humphreys K, Czene K, Moss S, et al. Comparison of fully and semi-automated area-based methods for measuring mammographic density and predicting breast cancer risk. Br J Cancer. 2014;110:1908-16.

16. Li J, Szekely L, Eriksson L, Heddson B, Sundbom A, Czene K, et al. High-throughput mammographic-density measurement: a tool for risk prediction of breast cancer. Breast Cancer Res. 2012;14:R114.

17. Couwenberg AM, Verkooijen HM, Li J, Pijnappel RM, Charaghvandi KR, Hartman M, et al. Assessment of a fully automated, high-throughput mammographic density measurement tool for use with processed digital mammograms. Cancer Causes Control. 2014;25:1037-43.

18. Michailidou K, Hall P, Gonzalez-Neira A, Ghoussaini M, Dennis J, Milne RL, et al, Large-scale genotyping identifies 41 new loci associated with breast cancer risk. Nat Genet. 2013;45:353-61. 361e1-2.

19. Purcell $S$, Neale B, Todd-Brown $K$, Thomas $L$, Ferreira MA, Bender D, et al PLINK: a tool set for whole-genome association and population-based linkage analyses. Am J Hum Genet. 2007:81:559-75.

20. 1000 Genomes Project Consortium, Abecasis GR, Auton A, Brooks LD, DePristo MA, Durbin RM, et al. An integrated map of genetic variation from 1,092 human genomes. Nature. 2012;491:56-65.

21. Howie B, Fuchsberger C, Stephens M, Marchini J, Abecasis GR. Fast and accurate genotype imputation in genome-wide association studies through pre-phasing. Nat Genet. 2012;44:955-9.

22. Willer CJ, Li Y, Abecasis GR. METAL: fast and efficient meta-analysis of genome-wide association scans. Bioinformatics. 2010;26:2190-1.

23. Pruim RJ, Welch RP, Sanna S, Teslovich TM, Chines PS, Gliedt TP, et al. LocusZoom: regional visualization of genome-wide association scan results. Bioinformatics. 2010;26:2336-7.

24. Marchini J, Howie B, Myers S, McVean G, Donnelly P. A new multipoint method for genome-wide association studies by imputation of genotypes. Nat Genet. 2007;39:906-13.

25. Marchini J, Howie B. Genotype imputation for genome-wide association studies. Nat Rev Genet. 2010;11:499-511.

26. Price AL, Patterson NJ, Plenge RM, Weinblatt ME, Shadick NA, Reich D. Principal components analysis corrects for stratification in genome-wide association studies. Nat Genet. 2006:38:904-9.

27. Patterson N, Price AL, Reich D. Population structure and eigenanalysis. PLoS Genet. 2006;2:e190.

28. Ward LD, Kellis M. HaploReg: a resource for exploring chromatin states, conservation, and regulatory motif alterations within sets of genetically linked variants. Nucleic Acids Res. 2012;40:D930-4. 
29. ENCODE Project Consortium. An integrated encyclopedia of DNA elements in the human genome. Nature. 2012;489:57-74.

30. Ernst J, Kheradpour P, Mikkelsen TS, Shoresh N, Ward LD, Epstein CB, et al. Mapping and analysis of chromatin state dynamics in nine human cell types. Nature. 2011:473:43-9.

31. Long J, Cai Q, Sung H, Shi J, Zhang B, Choi JY, et al. Genome-wide association study in east Asians identifies novel susceptibility loci for breast cancer. PLoS Genet. 2012;8:e1002532.

32. Zheng W, Zhang B, Cai Q, Sung H, Michailidou K, Shi J, et al. Common genetic determinants of breast-cancer risk in East Asian women: a collaborative study of 23637 breast cancer cases and 25579 controls. Hum Mol Genet. 2013;22:2539-50.

33. Takaesu G, Kishida S, Hiyama A, Yamaguchi K, Shibuya H, Irie K, et al. TAB2, a novel adaptor protein, mediates activation of TAK1 MAPKKK by linking TAK1 to TRAF6 in the IL-1 signal transduction pathway. Mol Cell. 2000;5:649-58.

34. Benson JR. Role of transforming growth factor beta in breast carcinogenesis. Lancet Oncol. 2004;5:229-39.

35. Barcellos-Hoff MH, Akhurst RJ. Transforming growth factor-beta in breast cancer: too much, too late. Breast Cancer Res. 2009;11:202.

36. Lee E, Van Den Berg D, Hsu C, Ursin G, Koh WP, Yuan JM, et al. Genetic variation in transforming growth factor beta 1 and mammographic density in Singapore Chinese women. Cancer Res. 2013;73:1876-82.

37. Zhu P, Baek SH, Bourk EM, Ohgi KA, Garcia-Bassets I, Sanjo H, et al. Macrophage/cancer cell interactions mediate hormone resistance by a nuclear receptor derepression pathway. Cell. 2006;124:615-29.

38. Cutrupi S, Reineri S, Panetto A, Grosso E, Caizzi L, Ricci L, et al. Targeting of the adaptor protein Tab2 as a novel approach to revert tamoxifen resistance in breast cancer cells. Oncogene. 2012;31:4353-61.

39. Wang $Y$, He $Y$, Qin Z, Jiang $Y$, Jin $G, M a ~ H$, et al. Evaluation of functional genetic variants at $6 \mathrm{q} 25.1$ and risk of breast cancer in a Chinese population. Breast Cancer Res. 2014;16:422.

40. Zheng W, Long J, Gao YT, Li C, Zheng Y, Xiang YB, et al. Genome-wide association study identifies a new breast cancer susceptibility locus at 6q25.1. Nat Genet. 2009;41:324-8.

41. Yang Z, Shen J, Cao Z, Wang B. Association between a novel polymorphism (rs2046210) of the 6q25.1 locus and breast cancer risk. Breast Cancer Res Treat. 2013:139:267-75.

42. Veeraraghavan J, Tan Y, Cao XX, Kim JA, Wang X, Chamness GC, et al. Recurrent ESR1-CCDC170 rearrangements in an aggressive subset of oestrogen receptor-positive breast cancers. Nat Commun. 2014;5:4577.

43. Assi V, Warwick J, Cuzick J, Duffy SW. Clinical and epidemiological issues in mammographic density. Nat Rev Clin Oncol. 2011;9:33-40.

44. Cheddad A, Czene K, Eriksson M, Li J, Easton D, Hall P, et al. Area and volumetric density estimation in processed full-field digital mammograms for risk assessment of breast cancer. PLoS One. 2014;9:e110690.

45. Stone J, Thompson DJ, Dos Santos Silva I, Scott C, Tamimi RM, Lindstrom S, et al. Novel associations between common breast cancer susceptibility variants and risk-predicting mammographic density measures. Cancer Res. 2015. doi:10.1158/0008-5472.

\section{Submit your next manuscript to BioMed Central and take full advantage of:}

- Convenient online submission

- Thorough peer review

- No space constraints or color figure charges

- Immediate publication on acceptance

- Inclusion in PubMed, CAS, Scopus and Google Scholar

- Research which is freely available for redistribution 